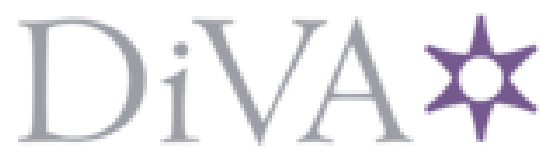

http://www.diva-portal.org

This is the published version of a paper published in IEEE Geoscience and Remote Sensing Letters.

Citation for the original published paper (version of record):

Kusetogullari, H., Yavariabdi, A. (2017)

Change Detection in Multispectral Landsat Images Using Multiobjective Evolutionary

Algorithm.

IEEE Geoscience and Remote Sensing Letters, 14(3): 414-418

https://doi.org/10.1109/LGRS.2016.2645742

Access to the published version may require subscription.

N.B. When citing this work, cite the original published paper.

Permanent link to this version:

http://urn.kb.se/resolve?urn=urn:nbn:se:bth-13978 


\title{
Change Detection in Multispectral Landsat Images Using Multiobjective Evolutionary Algorithm
}

\author{
Amir Yavariabdi and Huseyin Kusetogullari, Member, IEEE
}

\begin{abstract}
In this letter, we propose a novel method for unsupervised change detection in multitemporal multispectral Landsat images using multiobjective evolutionary algorithm (MOEA). The proposed method minimizes two different objective functions using MOEA to provide tradeoff between each other. The objective functions are used for evaluating changed and unchanged regions of the difference image separately. The difference image is obtained by using the structural similarity index measure method, which provides combination of the comparisons of luminance, contrast, and structure between two images. By evolving a population of solutions in the MOEA, a set of Pareto optimal solution is estimated in a single run. To find the best solution, a Markov random field fusion approach is used. Experiments on semisynthetic and real-world data sets show the efficiency and effectiveness of the proposed method.
\end{abstract}

Index Terms-Change detection, image processing, Landsat images, multiobjective evolutionary algorithms (MOEAs), remote sensing.

\section{INTRODUCTION}

$\mathbf{I}_{\mathrm{r}}^{\mathrm{N}}$ $\mathrm{N}$ REMOTE sensing context, change detection is the process of identifying differences between coregistered images acquired in the same geographical area at different times. This is an important research area as the time and precision of change detection on the land surface allow for deeper comprehension of the interactions between human and natural phenomena and can provide guidance in the management of the use of lands and resources [1]. Satellite imageries-for instance, synthetic aperture radar (SAR) and remotely sensed optical (e.g., Landsat) — have long been used to view and monitor land surface conditions, owing to their frequency of data collections and spatial resolutions. The SAR images are obtained using active sensors, which are working based on detection of the energy reflected from the Earth. Thus, this imagery is not sensitive to any meteorologic conditions. On the other hand, multispectral satellite images are acquired via passive optical sensors, and thereby, this remote sensing technique is sensitive to even very small changes in atmospheric conditions [2].

In general, change detection methods can be classified into three main categories: supervised [3], semisupervised [4], and unsupervised [5], [6] approaches. In the supervised and semisupervised approaches, a training set with accurate ground

Manuscript received March 17, 2016; revised July 27, 2016, September 28, 2016, and December 8, 2016; accepted December 20, 2016. The work of H. Kusetogullari was supported by the Research Project "Scalable resource efficient systems for big data analytics" funded by the Knowledge Foundation, Sweden, under Grant 20140032.

A. Yavariabdi is with the Department of Mechatronic Engineering, Karatay University, 42020 Konya, Turkey (e-mail: amir.yavari@gmail.com).

H. Kusetogullari is with the Department of Computer Science and Engineering, Blekinge Institute of Technology, 37141 Karlskrona, Sweden (e-mail: hku@bth.se).

Color versions of one or more of the figures in this letter are available online at http://ieeexplore.ieee.org.

Digital Object Identifier 10.1109/LGRS.2016.2645742 truth must be used to calculate the transformation function via a classifier, which will be used to flag each pixel as changed or unchanged. However, in satellite change detection context, generating accurate ground truth information is usually a tedious, difficult, and expensive task. Therefore, many unsupervised change detection methods have been proposed as there is no need of training sets. In general, the unsupervised change detection methods fall into two discrete groups: pixelbased and object-based change detection. In the pixel-based approach, an image pixel is used as fundamental unit of analysis whereas in the object-based approach, first, the object must be segmented or detected from images, and then its characteristics, such as shape, texture, topological information, and spectral response, can be used for further analysis [7]. Choosing appropriate unsupervised change detection approach is highly dependent on the objectivity of the research as pixel-based change detection methods are mostly suitable for low- and medium-resolution satellite images and object-based change detection methods are appropriate for very high resolution image [7]. Here, we propose a pixel-based unsupervised change detection method for medium resolution data.

In general, the pixel-based unsupervised change detection algorithms include two steps: 1) comparison of two images and 2) image analysis. In the first step, the similarity between images is computed using an appropriate mathematical model, such as: image differencing, log-ratio, change vector analysis (CVA), correlation coefficient (CC), erreur relative globale adimensionnelle de synthese (ERGAS), and so on. The second step is used to obtain binary change mask, which can be achieved by optimization methods [5], [6], thresholding [8], clustering approach [9], and many others [10]. For instance, Ghosh et al. [11] propose an unsupervised change detection technique for multispectral remote sensing images. Initially, a difference image is generated using the CVA [12]. Then, the difference image is modeled with Markov random field (MRF) and the binary change mask is estimated using the maximum a posteriori probability estimation criterion. The main drawback of this method is that the CVA similarity measure is based on pixelwise radiometric comparison, which makes it highly dependent of a radiometric correction method. This simply limits the application of their method in Landsat imaging. Renza et al. [8] propose an unsupervised method for change detection in multispectral remote sensing data. In this method, a difference image is obtained via the ERGAS similarity metric. After that, different thresholding algorithms are used to generate the final binary change mask and the best result is achieved using Otsu's thresholding algorithm. This similarity metric has a good sensitivity to global spectral distortion but it is sensitivity to local color distortion [13]. Therefore, this framework may provide poor performance for Landsat images, especially, when they captured with different atmospheric conditions. Celik [5] proposes an unsupervised change detection on the difference image by using a genetic 
algorithm (GA). The difference image is obtained using the log-ratio operator. The method minimizes a cost function using GA to generate the final change detection mask. However, the log-ratio is highly sensitive to a small number of pixels that have intensity differences between two images. In general, these algorithms share two common issues such as: 1) the cost functions are based on weighted sum of the changed and unchanged objectives and 2) the methods cannot cope with the phenomenon of atmospheric changes and/or speckle noise. Note that the first issue may cause high error detection rate as improvement of one objective function may cause deterioration for another objective function during the optimization process; hence, the change detection methods based on single cost function may not provide optimal tradeoff between changed and unchanged objective functions. To solve the second issue in change detection problem for SAR imaging, which often suffers from speckle noise, pixel-based multiobjective clustering-based change detection methods have been proposed [14]. However, in the context of change detection in Landsat images, these methods cannot be used as they are not robust to atmospheric conditions (e.g., the presence of haze, fog, smoke, or thin cloud), which can reduce the contrast of the observed area and fade the colors in the image.

In this letter, the structural similarity index measure (SSIM) [15] is used to measure the similarity between two Landsat images, based on local luminance, contrast, and structure comparisons. It has been used in various applications, such as image compression [15], video surveillance [16], object detection [3], and so on. To the best of our knowledge, the SSIM has not been used for the purpose of satellite change detection. The main reason of using the SSIM for change detection in Landsat images is to make the proposed method robust to contrast, brightness, and local color distortions [16] as well as haze and thin cloud. After that, two cost functions based on the SSIM for changed and unchanged pixels have been generated. Unlike the other unsupervised change detection methods, which are using weighted sum of the objective functions, we iteratively minimize two objective functions independently and simultaneously using multiobjective evolutionary algorithm (MOEA) to obtain a set of multiple binary change masks with the minimum influence of atmospheric conditions. More specifically, the MOEA provides the optimum tradeoff between the objective functions for better discrimination. Finally, MRF fusion technique [17] is adapted to optimally fuse the obtained binary masks for finding the final binary image. The experimental results on both synthetic with the existence of haze and thin cloud and real data sets show the effectiveness and robustness of the proposed method over the state-of-the-art methods.

The rest of this letter is organized as follows. The proposed method is described in Section II. Section III provides the experimental results of the proposed method and the state-ofthe-art methods. This letter is concluded in Section IV.

\section{Proposed Change Detection Method}

Assume $X_{1}^{(n)}$ and $X_{2}^{(n)}$, where $n=\{1,2,3\}$ denotes spectral bands, be two Landsat images with a size of $H \times W$. The main important objective of this letter is to find binary change detection image $X_{b}: \Omega \subset \mathbb{Z}^{2} \rightarrow\{0,1\}$, where the image domain $\Omega=\{1, \ldots, H\} \times\{1, \ldots, W\}$. By convention, 0 indicates that there is no change for the corresponding pixel and 1 indicates that there is a change.
In pixelwise change detection problem, obtaining the optimum binary image is a very challenging task as it has $2^{H \times W} \times n$ possible solutions. In order to obtain the optimal solution, it is necessary to use an optimization approach. In this letter, we propose a new unsupervised method in satellite images using MOEA. In this manner, the change detection problem can be treated as unsupervised binary classification task, which may require generation of difference image $X_{d}^{(n)}$ beforehand. To this end, the proposed method mainly consisting of three steps.

1) SSIM method is used as a similarity metric to compute the difference image. The main advantage of the SSIM operator compared with the frequency-used similarity metrics, i.e., image differencing, log-ratio, and $\mathrm{CC}$, is that the existing methods estimate perceived errors to quantify image differences, while the SSIM method considers image differences as perceived changes of structural information.

2) Two mean square error (MSE)-based cost functions are proposed to quantify changed and unchanged pixels. Then, the cost functions are separately optimized using MOEA to generate a set of optimal binary masks.

3) The MRF fusion is used to find the best solution.

\section{A. Landsat Image Similarity Measures via SSIM}

In the first step, the difference image must be generated. When detecting changes in optical images, the common way of comparing a pair of images is to compute a difference image using image subtraction. However, when Landsat images are considered, this technique is error prone as it is not robust to contrast, brightness, and color differencing changes. Therefore, we attempt to use SSIM as a difference measure technique, because it is effective when there are some differences between two images in intensity $s$, brightness $l$, and contrast $c$. The SSIM technique uses three components: luminance, contrast, and structure [15]. The SSIM can be calculated as follows:

$X_{d}^{(n)}=\left[l\left(X_{1}^{(n)}, X_{2}^{(n)}\right)\right]^{\alpha}\left[c\left(X_{1}^{(n)}, X_{2}^{(n)}\right)\right]^{\beta}\left[s\left(X_{1}^{(n)}, X_{2}^{(n)}\right)\right]^{\gamma}$

where, $\alpha, \beta$, and $\gamma$ are the exponents and used to adjust the influence of each measurement on the final calculation of $X_{d}$. For each channel, $X_{d}$ is measured in the same manner proposed in [15], with $\alpha=\beta=\gamma=1$. In (1), the variable $l$, $c$, and $s$ can be written as

$$
\left\{\begin{array}{l}
l\left(X_{1}^{(n)}, X_{2}^{(n)}\right)=\frac{2 \mu_{X_{1}^{(n)}} \mu_{X_{2}^{(n)}}}{\mu_{X_{1}^{(n)}}+\mu_{X_{2}^{(n)}}+\epsilon_{1}} \\
c\left(X_{1}^{(n)}, X_{2}^{(n)}\right)=\frac{2 \sigma_{X_{1}^{(n)}} \sigma_{X_{2}^{(n)}}}{\sigma_{X_{1}^{(n)}}+\sigma_{X_{2}^{(n)}}+\epsilon_{2}} \\
s\left(X_{1}^{(n)}, X_{2}^{(n)}\right)=\frac{\sigma_{X_{1}^{(n)} X_{2}^{(n)}}}{\sigma_{X_{1}^{(n)}} \sigma_{X_{2}^{(n)}}+\epsilon_{3}}
\end{array}\right.
$$

where $\mu, \sigma$, and $\sigma_{X_{1} X_{2}}$ are the mean, standard deviation, and cross correlation (covariance) between two images, respectively, and $\epsilon_{1}, \epsilon_{2}$, and $\epsilon_{3}$ are small positive constants included to avoid instability. Note that (2) is the normalization step of the proposed method and is calculated based on normalized values. Unlike the image differencing, which is measured at the global level, the SSIM is computed locally. In this letter, the image statistics $\left(\mu, \sigma\right.$, and $\left.\sigma_{X_{1} X_{2}}\right)$ are computed in the way proposed in [15]. Here, we use a $15 \times 15$ circular symmetric Gaussian weighting function with standard deviation of 1.8 samples. In this way, $X_{d}$ illustrates a locally isotropic property. 


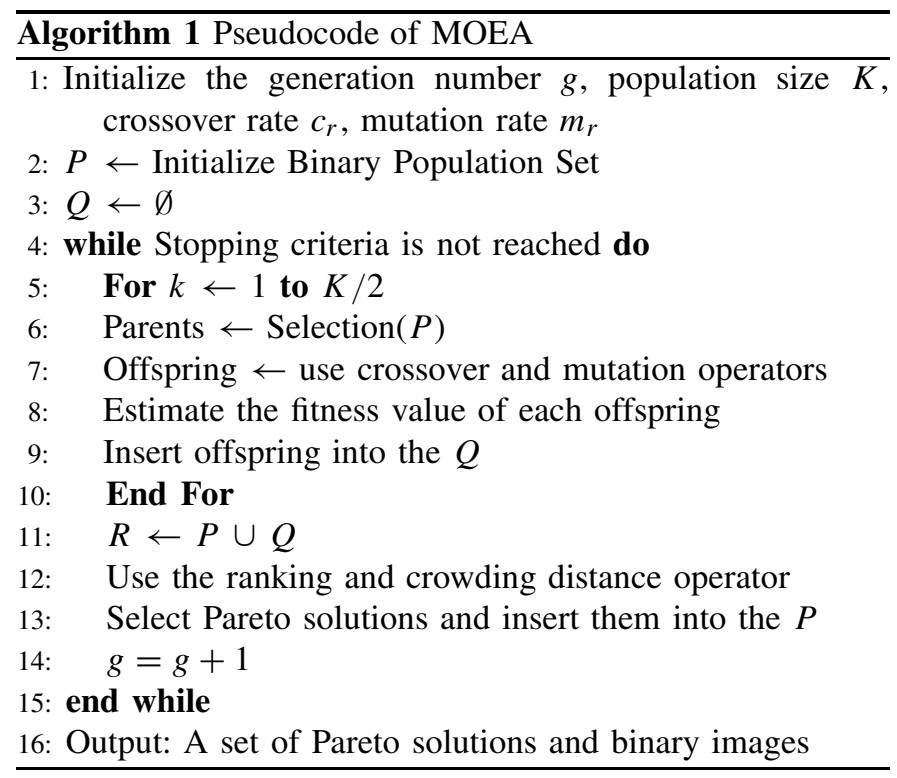

\section{B. Multiobjective Evolutionary Algorithm}

Many optimization problems contain multiple objectives and it is important to provide tradeoff between all objectives for obtaining an efficient result. A multiobjective optimization problem can be described as

$$
\text { minimize } F(x)=\left(f_{1}(x) f_{2}(x) \cdots f_{k}(x)\right)^{T}
$$

where $F(x)$ has $k$ objective functions and $x=\left[x_{1}, \ldots, x_{m}\right]$ is the vector of decision variables. To solve this optimization problem, one of the popular procedure is to transform multiobjective optimization problem into a single optimization function, and then, a single objective optimization algorithm (e.g., GA) can be used to find the optimal solution. However, this technique restricts the search space, so that improvement of one objective may cause deterioration for another objective during its iteration process. Thus, it may cause wrong decision making and decreasing accuracy rate. Recently, MOEAs have become great approaches to resolve multiple objectives simultaneously to provide the best tradeoff solutions between multiple objectives [18]. In the change detection context, there are several objective functions, which must be taken into account separately in order to find the changed and unchanged pixels more effectively. To resolve it, two objective functions $(k=2)$ are employed independently by using MOEA. Thus, Pareto optimal solutions are obtained as the best tradeoff solutions between the objectives. Edgeworth and Pareto [19] first presented the Pareto optimality approach and the method was used in [18]. Let $a, b \in \mathbb{R}^{k}$ be two different vectors and $a$ dominates $b$ (denoted by $a \prec b$ ) if $a_{z} \leq b_{z}$ for $z=1, \ldots, k$. On the other hand, $a$ and $b$ vectors are nondominated if $a_{j} \geq b_{j}$ for $j=1, \ldots, k$.

The purpose of the proposed method is to determine the set of Pareto optimal solutions. In this letter, we adopt the elitist nondominated sorting GA known as NSGA-II [20] to resolve the problem. In the NSGA-II, a number of chromosomes or individuals are randomly generated with the size of $H \times W$ for each spectral band and inserted in the population $P$. In each iteration, two chromosomes are randomly chosen from the population $P$ as parents by using the binary tournament selection operator and new individuals are produced by using the crossover and mutation operators, respectively. Crossover operates on two chromosomes and creates offspring by combining genes of both chromosomes. Thus, there is a transfer of genes between the parents, which leads to find better result. Mutation operator follows crossover and produces random changes of genes in various offspring to avoid getting stuck in a local optimum. Obtained new individuals are added in the new population $Q$. Then, both the current $P$ and the new population $Q$ are joined; the resulting population, $R$, is ordered according to Pareto solutions of chromosomes by using ranking operator and a density estimator known as crowding distance [20]. Thus, nondominated and dominated solutions are evaluated and the nondominated set of solutions are inserted into the population $P$. These steps are repeated until the termination criteria are satisfied. The pseudocode of the MOEA is given in Algorithm 1. Each chromosome in each iteration contains two different fitness values. To solve the multiobjective change detection problem using the NSGA-II, it is necessary to estimate fitness values of each chromosome. To achieve this, two objective functions are used to estimate fitness values of the candidates and these objective functions are formulated as follows:

$$
\begin{aligned}
C_{i}^{(n)}=\frac{N_{i}^{(n)}}{H \times W} \sum_{y=1}^{H} & \sum_{x=1}^{W}\left(\delta_{A_{i}}(x, y)\left(X_{d}^{(n)}(x, y)\right)\right. \\
& \left.-\frac{1}{N_{i}^{(n)}} \sum_{y=1}^{H} \sum_{x=1}^{W} \delta_{A_{i}}(x, y)\left(X_{d}^{(n)}(x, y)\right)\right)^{2}
\end{aligned}
$$

where $i=\{0,1\}, C_{0}^{(n)}$ and $C_{1}^{(n)}$ are two different fitness functions based on unchanged and changed pixels $\left(A_{0}\right.$ and $\left.A_{1}\right)$ at the $n$th spectral band, respectively, and $N_{0}^{(n)}$ and $N_{1}^{(n)}$ are the number of changed and unchanged pixels on the change detection mask, respectively. $\delta_{A_{1}}(x, y)$ is the Kronecker delta function whose value is 1 at $\forall(x, y) \in A_{1}$ and 0 otherwise whereas $\delta_{A_{0}}(x, y)$ is 1 at $\forall(x, y) \in A_{0}$ and 0 otherwise.

In (4), the objective functions are computed based on the MSE of their difference image values $\left(X_{d} \in A_{i}\right)$ and the mean of their difference image values. Note that the lower the MSE, the better the detection result is. The common way to achieve this is to estimate weighted sum of the MSEs of the unchanged and changed areas and minimize it. However, this objective function cannot grantee an optimal solution as indeed giving the possibility to decrease one of the MSEs and to increase the other. To solve this problem, we separately calculate the objective functions (4) for changed and unchanged areas and optimize them simultaneously using the NSGA-II. The proposed method provides multioptimum change detection masks (a set of Pareto optimal solutions). In order to find the final binary mask, it is necessary to merge all the solutions. To this end, Chen et al. [17] propose a fusion approach based on MRF to merge two binary masks, and here, their equation of the Markov modeling of the conditional distribution of the pixel label is adapted to fuse $m$ binary masks.

\section{EXPERIMENTAL RESULTS}

The accuracy of the proposed method is evaluated by quantitative and qualitative tests on various semisynthetic images and two real medium resolution Landsat data sets. In realdata experiments, the first data set with $1984 \times 1451$ pixels shows the water surface of the lake Milh (Iraq) in 1995 and 2003 [Fig. 1(a) and (b)]. For more comprehensive quantitative 


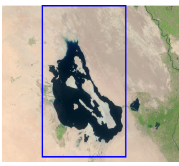

(a)

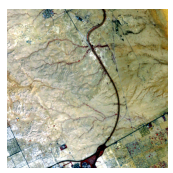

(e)

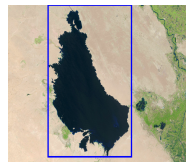

(b)

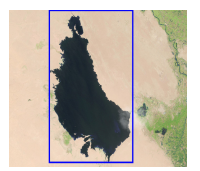

(c)

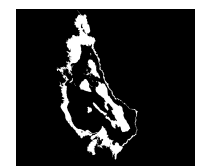

(d)

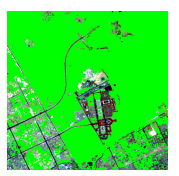

(g)
Fig. 1. (a) and (b) Lake Milh data set. (c) Image with artificial haze and thin cloud. (d) Ground truth change mask for region of interest (blue box). (e) and (f) Princess Nora Bint Abdul Rahman University images. (g) Changed and unchanged (green) pixels detected by the proposed method for (e) and (f).

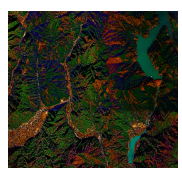

(a)

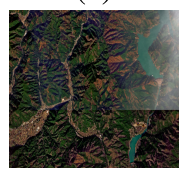

(e)

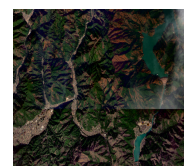

(b)

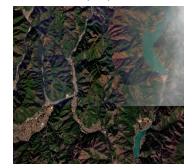

(f)

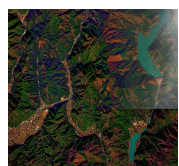

(c)

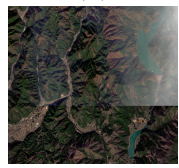

(g)

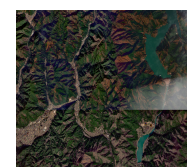

(d)
Fig. 2. Example of generated semisynthetic data. (a) Reference image $\left(X_{1}\right)$. (b)-(g) $X_{2}$ images, which cover by increasingly larger levels of haze and thin clouds.

evaluations, the haze and thin cloud are artificially added to Fig. 1(b) and it is shown in Fig. 1(c). For this data set, the ground truth change mask is generated by manually labeling of region of interest (blue box) and shown in Fig. 1(d). In addition, the region of interest is considered for conducting quantitative evaluations. Fig. 1(e) and (f) shows the Princess Nora Bint Abdul Rahman university in north of Riyadh and they are captured in 2003 and 2013 with the resolution of $396 \times 392$ pixels. Since the changes in land use have complicated patterns, it is not easy to generate the ground truth. Therefore, to make the qualitative evaluation easier, the proposed method is used to obtain changed and unchanged pixels and then project the pixel information from Fig. 1(g) into changed regions and green color into unchanged areas.

The proposed method is compared with EM-based [21], GA-based [5], ERGAS-based [8], and Particle Swarm Optimization (PSO)-GA-based [6] change detection methods. The proposed method, PSO-GA-based, and ERGAS-based methods use the images in RGB color space whereas the Expectation Maximization (EM)-based and GA-based methods use gray-scale images. The EM-based and ERGAS-based methods do not have any parameter to set. In other two methods, we use the parameters given in [6]. In the proposed approach, the NSGA-II is used with the population size of 30 , the crossover rate of 0.8 , the mutation rate of 0.01 , and the iteration number of 25000 . These parameters are selected empirically. Besides the qualitative tests, the change detection accuracies are evaluated using some standardized measures, including false alarm rate $\left(P_{\mathrm{FA}}\right)$, missed detection rate $\left(P_{\mathrm{MD}}\right)$, and total error $\left(P_{\mathrm{TE}}\right)[6]$.

It is important to evaluate the robustness of the algorithms on various atmospheric conditions, such as thin clouds
TABLE I

Quantitative Results For Semis ynthetic Data Sets

\begin{tabular}{|c||c|c|c|c|c|c|}
\hline \multirow{2}{*}{ Method } & \multicolumn{7}{|c|}{ Data sets from Fig. 2} \\
\cline { 2 - 7 } & I:(a)-(b) & II:(a)-(c) & III:(a)-(d) & IV:(a)-(e) & V:(a)-(f) & VI:(a)-(g) \\
\hline \hline EM-based & 2.7580 & 4.6118 & 5.7812 & 9.3199 & 14.0531 & 18.4795 \\
\hline GA-based & 0.9734 & 2.2525 & 5.4044 & 7.5438 & 12.2617 & 17.3370 \\
\hline PSO-GA-based & 0.1903 & 0.3213 & 1.1198 & 5.7790 & 9.0519 & 15.2318 \\
\hline ERGAS-based & 4.07 & 5.35 & 10.48 & 14.59 & 13.50 & 20.07 \\
\hline Proposed & 0.0305 & 0.09980 & 0.2307 & 1.3922 & 3.0533 & 6.0384 \\
\hline
\end{tabular}

TABLE II

Quantitative Results for Lake Milh Data Sets

\begin{tabular}{|c||c|c|c||c|c|c||}
\hline \multirow{2}{*}{ Method } & \multicolumn{3}{|c||}{ Lake Milh (Fig. 1 (a)-(b)) } & \multicolumn{3}{c|}{ Lake Milh (Fig. 1 (a)-(c)) } \\
\cline { 2 - 7 } & $P_{F A}$ & $P_{M A}$ & $P_{T E}$ & $P_{F A}$ & $P_{M A}$ & $P_{T E}$ \\
\hline \hline EM-based & 3.04 & 22.49 & 9.01 & 3.85 & 27.67 & 12.83 \\
\hline GA-based & 2.36 & 7.16 & 3.87 & 3.06 & 9.69 & 5.50 \\
\hline PSO-GA-based & 1.28 & 2.55 & 1.71 & 1.93 & 2.99 & 2.08 \\
\hline ERGAS-based & 2.50 & 4.35 & 1.97 & 16.22 & 4.23 & 7.76 \\
\hline Proposed & 1.09 & 0.53 & 0.90 & 1.52 & 0.62 & 1.26 \\
\hline
\end{tabular}

and haze by conducting experiments on semisynthetic data. To achieve this, an image covered by haze and thin cloud [see Fig. 2(g)] is processed by a visibility restoration algorithm based on filtering approach [22] to partially or fully remove haze and thin cloud. In this manner, we generate six semisynthetic images, which are shown in Fig. 2(a)-(f). In the change detection algorithms, Fig. 2(a) is considered as $X_{1}$, since it is the result for clear weather, whereas each image in Fig. 2(b)-(g) is considered as $X_{2}$. Since there is no land changed on the observed area, the ground truth of the change map does not include any changed pixel, so that the $P_{F A}$ is used as a quantitative measurement.

The quantitative results for the data sets which are shown in Fig. 2 are tabulated in Table I. The results show that the ERGAS-based and EM-based methods yield the highest rate of incorrect detections. The EM-based method always detects the haze and thin cloud region as changed. The ERGAS-based method shows high sensitivity to local color distortions, and due to the use of simple and noneffective thresholding algorithm for binarization of the difference image, many pixels are wrongly marked as changed. The GA-based method provides the higher accuracy than EM-based and ERGAS-based methods, because it uses an optimization strategy, but the results are far from being satisfactory, except for the data with very low level of haze and thin cloud. The main reason that the EM-based and GA-based methods detect haze, thin cloud, and color differences between two images as changed is because they are based on image differencing. The results of the PSO-GA method are a good evidence to show that using cross correlation, which is less sensitive than the image differencing to intensity variations between two images, can improve the performance of optimization-based change detection significantly. However, this method is robust to a certain amount of the intensity variations. For instance, Table I shows that the PSO-GA method can provide high accurate change detection results for data sets I-III; however, for more sophisticated atmospheric conditions, the precision and accuracy sharply decrease. Finally, by comparing the results, we can conclude that the proposed method provides the best results as the SSIM operator is strongly robust to intensity variations. In addition, MOEA provides tradeoff between changed and unchanged objective functions, which leads to the least $P_{F A}$, as shown in Table II.

To validate our method and to test its robustness to real environmental change, three data sets are used. For instance, we use a set of Landsat images showing urban growth [see Fig. 1(d) and (e)]. The results are shown in Fig. 3. Visually, 


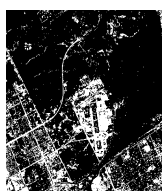

(a)

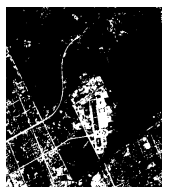

(b)

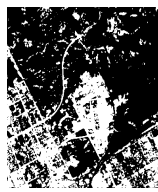

(c)

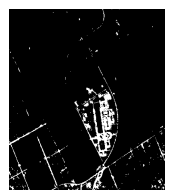

(d)

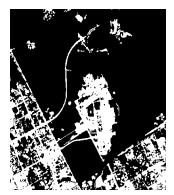

(e)
Fig. 3. Change detection results for the Land use data set. (a) EM. (b) GA. (c) PSO-GA. (d) ERGAS. (e) Proposed.

we can see that the ERGAS-based method is the least accurate and effective method among the four as Otsu's thresholding gives nonoptimal thresholding value. The EM-based method provides better result than ERGAS-based method, but it has still high failure rate. The GA-based method contains many isolated pixels and it flags the soil reflectance as changed pixels. These problems are solved using the PSO-GA and the proposed methods. However, the PSO-GA method has higher missed detection rate than the proposed method. Even though the proposed method shows good sensitivity to soil reflectance, it fails in the places where the soil has very high reflectance. Moreover, the quantitative results for the region of interest in the lake Milh data set with and without haze and thin cloud are tabulated in Table II. Using Fig. 1(a) and (b) shows that the proposed method obtains the least overall error of $0.90 \%$. According to the results in Table II for Fig. 1(a)-(c), it is seen that $P_{\mathrm{FA}}, P_{\mathrm{MA}}$, and $P_{\mathrm{TE}}$ slightly increase using the proposed method. On the other hand, the results of the compared methods show that the haze and thin cloud have large influence on their performances. As a result, the ERGAS-based and EM-based methods are not precise. The accuracy of change detection is slightly improved by using GA-based method at the cost of computational time. On the other hand, the PSO-GA and the MOEA provide more precise results while using less iterations.

The qualitative and quantitative tests show that the GA-based and the PSO-GA-based methods provide less accurate results as the multiobjective optimization problem is converted into a single optimization problem. In contrast, the proposed method obtains the change detection mask more accurate than the other methods. This is due to the fact that the proposed method is robust to contrast, brightness, and color inconsistency changes, so that it has very few isolated pixels and can correctly detect haze and thin cloud as unchanged. Despite the advantages of the proposed method, MOEA has a disadvantage, which is a time-consuming algorithm as the speed of iterations depends on the size of the input images and the population size. Thus, this method may suffer for large resolution images.

\section{CONCLUSION}

This letter has proposed a new MOEA-based change detection method for multispectral Landsat images. The proposed method uses two different objective functions based on SSIM operator, which provides combination of the comparisons of luminance, contrast, and structure between two images. The objective functions are iteratively minimized independently and separately using MOEA, which results in a set of optimal binary masks. Finally, for finding the best binary change detection mask, an MRF framework is used to optimally fuse the obtained binary masks. Experimental results on a semisynthetic data set with various levels of thin cloud and haze and two pairs of real Landsat images have demonstrated that the proposed approach is robust to atmospheric changes and achieves the best detection performance than other compared change detection methods.

\section{ACKNOWLEDGMENT}

The authors would like to thank Prof. T. Celik for sharing his codes.

\section{REFERENCES}

[1] F. A. Alqurashi and L. Kumar, "Investigating the use of remote sensing and GIS techniques to detect land use and land cover change: A review," Adv. Remote Sens., vol. 2, no. 2, pp. 193-204, 2013.

[2] G. A. Shaw and H. H. K. Burke, "Spectral imaging for remote sensing," Lincoln Lab. J., vol. 14, no. 1, pp. 3-28, 2003.

[3] G. Sarp and M. Ozcelik, "Water body extraction and change detection using time series: A case study of Lake Burdur, Turkey," J. Taibah Univ. Sci., to be published, doi: 10.1016/j.jtusci.2016.04.005.

[4] C. Wu, B. Du, and L. Zhang, "Slow feature analysis for change detection in multispectral imagery," IEEE Trans. Geosci. Remote Sens., vol. 52, no. 5, pp. 2858-2874, May 2014.

[5] T. Celik, "Change detection in satellite images using a genetic algorithm approach," IEEE Geosci. Remote Sens. Lett., vol. 7, no. 2, pp. 386-390, Apr. 2010.

[6] H. Kusetogullari and A. Yavariabdi, "Self-adaptive hybrid PSO-GA method for change detection under varying contrast conditions in satellite images," in Proc. IEEE Int. Conf. Sci. Inf. Comput., Jul. 2016, pp. 361-368.

[7] M. Hussain, D. Chen, A. Cheng, H. Wei, and D. Stanley, "Change detection from remotely sensed images: From pixel-based to objectbased approaches," ISPRS J. Photogram. Remote Sens., vol. 80, pp. 91-106, Jun. 2013.

[8] D. Renza, E. Martinez, and A. Arquero, "A new approach to change detection in multispectral images by means of ERGAS index," IEEE Geosci. Remote Sens. Lett., vol. 10, no. 1, pp. 76-80, Jan. 2013.

[9] Y. Zheng, X. Zhang, B. Hou, and G. Liu, "Using combined difference image and $k$-means clustering for SAR image change detection," IEEE Geosci. Remote Sens. Lett., vol. 11, no. 3, pp. 691-695, Mar. 2014.

[10] G. Jianya, S. Haigang, M. Guorui, and Z. Qiming, "A review of multitemporal remote sensing data change detection algorithms," Int. Arch. Photogram., Remote Sens. Spatial Inf. Sci., vol. 37, no. B7, pp. 757-762, 2008.

[11] A. Ghosh, B. N. Subudhi, and L. Bruzzone, "Integration of Gibbs Markov random field and Hopfield-type neural networks for unsupervised change detection in remotely sensed multitemporal images," IEEE Trans. Image Process., vol. 22, no. 8, pp. 3087-3096, Aug. 2013.

[12] R. J. Radke, S. Andra, O. Al-Kofahi, and B. Roysam, "Image change detection algorithms: A systematic survey," IEEE Trans. Image Process., vol. 14, no. 3, pp. 294-307, Mar. 2005.

[13] F. Samadzadegan and F. DadrasJavan, "Evaluating the sensitivity of image fusion quality metrics to image degradation in satellite imagery," J. Indian Soc. Remote Sens., vol. 39, no. 4, pp. 431-441, 2011.

[14] H. Li, M. Gong, Q. Wang, J. Liu, and L. Su, "A multiobjective fuzzy clustering method for change detection in SAR images," Appl. Soft Comput., vol. 46, pp. 767-777, Sep. 2016.

[15] Z. Wang, A. C. Bovik, H. R. Sheikh, and E. P. Simoncelli, "Image quality assessment: From error visibility to structural similarity," IEEE Trans. Image Process., vol. 13, no. 4, pp. 600-612, Apr. 2004.

[16] A. Łoza, L. Mihaylova, D. Bull, and N. Canagarajah, "Structural similarity-based object tracking in multimodality surveillance videos," Mach. Vis. Appl., vol. 20, no. 2, pp. 71-83, 2009.

[17] K. Chen, C. Huo, J. Cheng, Z. Zhou, and H. Lu, "Unsupervised change detection on SAR images using Markovian fusion," Proc. SPIE, vol. 6790, p. 67901S, Nov. 2007.

[18] A. Zhou, B.-Y. Qu, H. Li, S.-Z. Zhao, P. N. Suganthan, and Q. Zhang, "Multiobjective evolutionary algorithms: A survey of the state of the art," Swarm Evol. Comput., vol. 1, no. 1, pp. 32-49, 2011.

[19] W. Stadler, "A survey of multicriteria optimization or the vector maximum problem, part I: 1776-1960," J. Optim. Theory Appl., vol. 29, no. 1, pp. 1-52, 1979.

[20] K. Deb, A. Pratap, S. Agarwal, and T. Meyarivan, "A fast and elitist multiobjective genetic algorithm: NSGA-II," IEEE Trans. Evol. Comput., vol. 6, no. 2, pp. 182-197, Apr. 2002.

[21] L. Bruzzone and D. F. Prieto, "Automatic analysis of the difference image for unsupervised change detection," IEEE Trans. Geosci. Remote Sens., vol. 38, no. 3, pp. 1171-1182, May 2000.

[22] J.-P. Tarel and N. Hautière, "Fast visibility restoration from a single color or gray level image," in Proc. IEEE Int. Conf. Comput. Vis., Sep./Oct. 2009, pp. 2201-2208. 